\title{
CONTROL DE RESIDUOS DE ANTIBIÓTICOS EN LECHE CRUDA EN UNA EMPRESA LECHERA EN JALISCO MÉXICO: ESTUDIO RETROSPECTIVO
}

\author{
Control of antibiotic residues in raw milk in a dairy industry from Jalisco, Mexico: a retrospective \\ study
}

\begin{abstract}
ras
Mario Noa- Pérez

Departamento de Salud Pública. Centro Universitario de Ciencias Biológicas y Agropecuarias, Universidad de Guadalajara. Camino Ramón Padilla Sánchez 2100 Nextipac, 45200 Zapopan, Jal. *Autor para correspondencia: mario.noa@academicos.udg.mx

Silvia Ruvalcaba- Barrera

Departamento de Desarrollo Rural. Centro Universitario de Ciencias Biológicas y Agropecuarias, Universidad de Guadalajara. Camino Ramón Padilla Sánchez 2100 Nextipac, 45200 Zapopan, Jal. José Pablo Torres- Morán

Departamento de Desarrollo Rural. Centro Universitario de Ciencias Biológicas y Agropecuarias, Universidad de Guadalajara. Camino Ramón Padilla Sánchez 2100 Nextipac, 45200 Zapopan, Jal.

Ramón Reynoso- Orozco

Departamento de Biología Celular y Molecular. Centro Universitario de Ciencias Biológicas y Agropecuarias, Universidad de Guadalajara. Camino Ramón Padilla Sánchez 2100 Nextipac, 45200 Zapopan, Jal.

Teresa de Jesús Jaime- Ornelas

Departamento de Salud Pública. Centro Universitario de Ciencias Biológicas y Agropecuarias, Universidad de Guadalajara. Camino Ramón Padilla Sánchez 2100 Nextipac, 45200 Zapopan, Jal.
\end{abstract}

\section{Resumen}

La normatividad vigente en México prohíbe la presencia de residuos de inhibidores de crecimiento microbiano, que incluyen los antibióticos y desinfectantes en leche cruda de vaca. Debido al impacto de la presencia de residuos de antibióticos en la leche sobre la elaboración de derivados lácteos, algunas empresas lecheras han incluido el control de residuos de antibióticos, para evitar la recolección de materia prima contaminada. El objetivo de este trabajo fue analizar los resultados de la aplicación de un programa de control de residuos de antibióticos asociado al control de calidad de la leche cruda en una empresa productora de derivados lácteos. Para ello se registró la frecuencia de detección en leche cruda de vaca de los dos principales grupos de antibióticos: $\beta$ - lactámicos y tetraciclinas, en nueve plantas enfriadoras de leche cruda de la empresa, con un volumen de recepción promedio total entre 800,000 y 1,000,000 L/día durante los primeros 5 años consecutivos a la introducción del programa. La detección de residuos se realizó utilizando los juegos de reactivos SNAP® Beta-Lactam Test and SNAP® Tetracycline Test simultáneamente. De 222 tanques conteniendo 2,955,538 L analizados en el primer año, 93 fueron positivos para un $42.11 \%$, pero ya en el quinto año de establecido el control, de 889 tanques $(5,658,062$ L) sólo 13 resultaron positivos $(1.46 \%)$, con una tasa de reducción promedio del $10.16 \%$ anual. Cuando la leche cruda es comprada por empresas que vigilan la calidad del producto, los ganaderos descartan la leche contaminada, sin embargo la venden con antibióticos cuando los compradores no realizan controles, por lo que ésta medida de control por parte de las empresas redunda en una solución. Los resultados mostraron una disminución significativa de la contaminación, siendo una solución útil al problema de la aparición de residuos de antibióticos en leche en la materia prima de la planta.

Palabras clave: Inhibidores, residuos en leche, control de calidad en leche.

\begin{abstract}
The current regulation in Mexico is explicit about the prohibition of the presence of antibiotic residues in raw cow's milk. Due to the impact of the presence of antibiotic residues in milk in the production of dairy products, some dairy companies have implemented their own programs to control antibiotic residues to avoid collection of contaminated material. The aim of this study was to analyze the results of the implementation of a program to control the presence of antibiotic residues in raw cow's milk collected by a big local dairy industry. During the applications of this program, the frequency of contaminated milk containing the two main groups of antibiotics: $\beta$-lactams and tetracyclines, in 9 concentration facilities of a local dairy company, with an average size receiving 800,000- 1,000,000 L/day during 5 years. The antibiotic residue detection was performed using SNAP® Beta-Lactam Test and SNAP® Tetracycline Test simultaneously. During the first year, 222 tanks
\end{abstract}


$(2,955,538 \mathrm{~L})$ were tested, of them 93 were positive for antibiotic residues $(42.11 \%)$. In the fifth year, from 889 tanks containing $5,658,062 \mathrm{~L}$ only 13 were positive $(1.46 \%)$, obtaining a reduction rate of $10.16 \%$ annual average. When the companies control the presence of inhibitor residues in the raw milk buy the milk, the farmers discard the contaminated milk; however, they sell it even with antibiotics when the buyers do not carry out controls, so this control measure becomes a solution. The results showed a significant decrease in contamination, being a useful solution to the problem of the appearance of antibiotic residues in milk in the raw material of the plant.

Key words: Inhibitors, residues in milk, quality control in milk.

\section{Introducción}

Jalisco es el Estado con mayor producción de leche en México: se producen actualmente alrededor de 2,400 millones de litros por año, lo que representa más del $20 \%$ de la producción nacional. El 55\% de esa producción, se destina a pasteurización (INEGI, 2019). Este volumen se distribuye en diversas industrias, algunas de las cuales se han preocupado por recolectar una materia prima que cumpla con todos los parámetros de calidad establecidos en la normatividad sanitaria (NOM-243, NMX-F-700).

La tarea de evitar residuos de antimicrobianos y otros fármacos en la leche se debe enfrentar desde varias perspectivas; en México, el asunto no ha dependido sólo de las autoridades, ya que los grandes consorcios comercializadores de leche de manera sistemática hacen pruebas para constatar que no haya en ésta residuos de sustancias antibacterianas (Sumano y Ocampo, 2006). Algunas empresas productoras de lácteos han implementado sus propios programas de control de residuos de antibióticos en la leche, como medida de control de calidad, con lo cual reducen considerablemente el problema de la aparición de sus residuos en la leche. Dicho programa de control comprende no sólo el monitoreo, sino la aplicación de medidas que van desde un castigo al precio de la leche cruda, hasta la suspensión de compra del producto al productor primario.

Es bien conocido que una vez presentes en la leche, los residuos de antibióticos no pueden ser eliminados empleando las vías normales de procesamiento industrial o doméstico (Kurjogi Mohammad, Alghamdi, Abdelrahman, Satapute et al, 2019; Roca, Villegas, Kortabitarte, Althaus, and Molina, 2011, Kellnerová, Navrátilová and Borkovcová, 2014). Entre los grupos de antimicrobianos que mayor número de formulaciones tienen registradas en México se encuentran los de las familias de $\beta$ - lactámicos y tetraciclinas, seguidos por las sulfonamidas (PLM, 2019). Los cultivos iniciadores utilizados en la producción de derivados lácteos fermentados, tales como queso y yogurt, son extremadamente sensibles a bajas concentraciones de antibióticos en la leche (Noa y Ruvalcaba, 2011), por lo cual una parte de las empresas procesadoras de lácteos han implementado sus propios programas de control de residuos de los los llamados inhibidores, con el fin de los llamados inhibidores, con el fin de reducir pérdidas económicas durante la compra de la leche cruda recibida.

El objetivo de este trabajo fue presentar los resultados de la aplicación de un programa de control de la presencia de residuos de antibióticos en leche cruda de vaca, acopiada por una empresa lechera, mediante el monitoreo de dos de las principales familias de antibióticos utilizadas en lechería ( $\beta$ lactámicos y tetraciclinas), durante el período los primeros cinco años de la aplicación del mismo.

\section{Materiales y métodos}

La información aquí presentada se recabó de una empresa lechera de Jalisco, que acopia entre 800,000 y un millón de litros de leche diarios. Los datos presentados corresponden a los primeros cinco años consecutivos de aplicación del programa de control de residuos de las dos principales familias químicas de antibióticos mediante una prueba grupo específica rápida, como parte del control higiénico- sanitario de la materia prima adquirida, y corresponden al análisis del volumen total de leche cruda de vaca captada por la empresa. Para éste fin, se analizaron los tanques de las nueve plantas receptoras y enfriadoras de leche cruda que recibe la empresa, destinadas a acopiar y analizar la materia prima para su posterior reenvío a la planta pasteurizadora. Estas enfriadoras cuentan con laboratorios propios donde se realiza un control de calidad primario de la leche acopiada. Este control incluye tanto parámetros físico- químicos (densidad, grasa, proteína, etc.), así como pruebas de calidad higiénico sanitarias como tiempo de reducción del azul de metileno, determinación de materia extraña, acidez, prueba del alcohol al $75 \%$, etc., antes de reenviar la materia prima a la pasteurizadora.

Con el objetivo de mejorar dicho control, se introdujo un método de prueba rápido (NMX-F-719-COFOCALEC-2008) de tipo grupo- específico para residuos de las dos familias de antibióticos más utilizadas en la ganadería lechera: antibióticos $\beta$ - lactámicos (penicilinas y cefalosporinas) y tetraciclinas.

Las plantas enfriadoras se ubican en sitios estratégicos del Estado de Jalisco, en las que confluye la leche procedente 
de las zonas de mayor producción.

La presencia de residuos de antibióticos en leche se analizó diariamente en cada carro- cisterna, previo al trasvase de la leche hacia el silo de la planta. Para los análisis se utilizaron los juegos de reactivos comerciales SNAP Beta- Lactam test ${ }^{\circledR}$ y SNAP Tetracycline Test ${ }^{\circledR}$, de IDEXX Laboratories, Inc. (Westbrook, Main, USA). El método, se describe brevemente como sigue:

Las pruebas SNAP Beta-Lactam y SNAP Tetracycline Test son ensayos tipo ELISA, que detectan residuos de antibióticos $\beta$ - lactámicos y tetraciclinas respectivamente, en pruebas separadas, en mezclas de leche cruda de vaca. La sensibilidad de estos juegos de reactivos logra alcanzar niveles de sensibilidad adecuados para detectar los LMR establecidos para México. El límite máximo de residuos (LMR) es la concentración máxima de residuos legalmente permitida en un producto alimenticio obtenido de un animal al que se le ha administrado un medicamento veterinario. Los resultados se interpretaron de forma cualitativa, o sea visualmente. Si el punto coloreado correspondiente a la muestra es igual de intenso o más oscuro que el punto de control del resultado, la muestra de leche se considera negativa a la familia de antibióticos correspondiente, de lo contrario se considera positiva.

Los valores de LMR para México (SADER- SENASICA, 2020) en leche se muestran en el Cuadro 2, conjuntamente con la sensibilidad del juego de reactivos utilizado en este estudio ("SNAP beta-lactam test” ${ }^{\circledR}$ y "SNAP tetracycline test" ${ }^{\circledR}$ (IIDEXX Laboratories, Inc) según las especificaciones declaradas por el fabricante (NMX-F-719-COFOCALEC2008), lo que garantiza la confiabilidad de los análisis realizados. Los resultados de frecuencia de detección fueron comparados estadísticamente mediante la prueba de Comparación Múltiple de Proporciones, incluida en el paquete estadístico SPSS. Ver. 12.0.

Cuadro 2. Sensibilidad de las pruebas SNAP® utilizadas en el programa de control de residuos de inhibidores y valor de LMR en leche.

\begin{tabular}{lcc}
\hline ANTIBIÓTICO & $\begin{array}{c}\text { SENSIBILIDAD }^{1} \\
\mu \mathrm{g} / \mathrm{L}\end{array}$ & $\begin{array}{c}\mathrm{LMR}^{2} \\
\mu \mathrm{g} / \mathrm{L}\end{array}$ \\
\hline Bencilpenicilina & 3 & 4 \\
Ampicilina & 5,8 & 10 \\
Amoxicilina & 7,3 & 10 \\
Cefapirina & 11,7 & 20 \\
Ceftiofur & 5,4 & 100 \\
Tetraciclina & 50 & \\
Clortetraciclina & 100 & 100 \\
Oxitetraciclina & 50 & \\
\hline Fuentes: ${ }^{2:}$ NMX-F-719-COFOCALEC-2008; & $2:$ SADER- \\
SENASICA, 2020 & &
\end{tabular}

\section{Resultados}

La implementación del programa de control de residuos fue paulatina. Durante el primer año se analizaron un promedio de 2,955,538 de litros de leche provenientes de 222 tanques, de los cuales 93 fueron positivos a residuos de antibióticos, representando un $42.11 \%$, en tanto cada año se incrementó paulatinamente el número de tanques analizados, hasta alcanzar 889 en el quinto año, como se muestra en la Figura 1 .

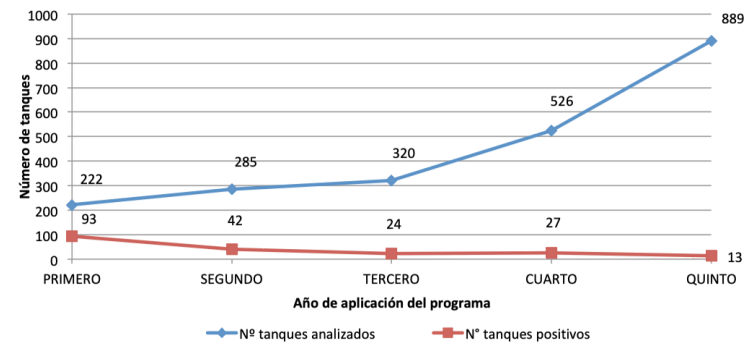

Figura 1. Número de tanques de leche analizados y positivos a residuos de antibióticos por año.

La primera respuesta a la detección positiva a residuos de antibióticos, una vez implementado el programa, fue castigar el precio de compra de la leche cruda, seguida por la separación de los productores reincidentes del padrón de acopiadores de la empresa, coincidiendo con los planteado ya desde 1991 por FIL/IDF . El porcentaje de positividad a residuos de antibióticos en el quinto año fue de $1.46 \%$, año en el que se evaluaron muestras representativas de 5,658,062 L en promedio, el número de tanques muestreados fue de 889 y únicamente 13 resultaron positivos (Figura 2), obteniéndose una tasa de reducción promedio anual del $10.16 \%$.

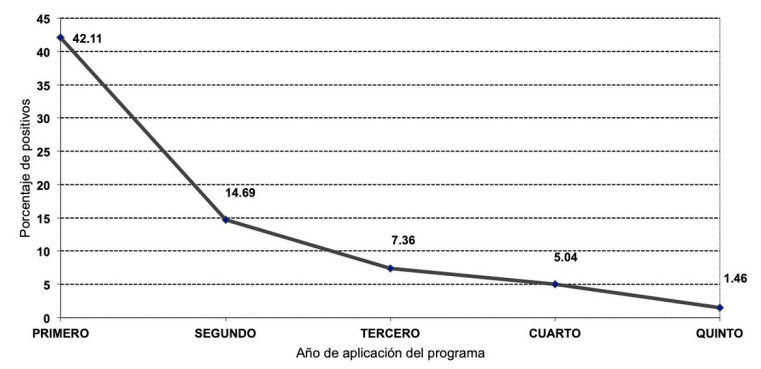

Figura 2. Frecuencia de muestras positivas a residuos de antibióticos por año

Esto significó una disminución significativa $(\mathrm{p}<0.05)$ en la frecuencia de tanques positivos a residuos de antibióticos, durante el período en que se realizó el presente estudio.

\section{Discusión}

La mastitis sigue siendo la primera causa del uso de antibacterianos en veterinaria, y la producción de leche con residuos es uno de los principales problemas de inocuidad en los alimentos. (Sumano y Ocampo, 2006). Los residuos de antimicrobianos en la leche pueden afectar la salud del consumidor, así como al proceso de manufactura de producto 
lácteos. Además, el calentamiento de la leche o su pasteurización no afectan la presencia de antibióticos; en todo caso sólo se modifica su actividad antimicrobiana (Sumano y Ocampo, 2006). La presencia de antimicrobianos en leche tiene especialmente un fuerte impacto sobre la industria láctea, ya que los cultivos iniciadores empleados en la producción de derivados lácteos fermentados, tales como queso y yogurt, son extremadamente sensibles a bajas concentraciones de sustancias inhibidoras en la leche (FIL/IDF, 1991).

En el presente estudio se puso de manifiesto que a partir del tercer año de aplicación del programa de control por parte de la empresa, se evidencia una disminución de la presencia de antibióticos, posiblemente debido a que los ganaderos comenzaron a asesorarse sobre la utilización de antibióticos y los tiempos de retiro. No obstante, existen factores externos, como la aplicación de preparados caseros que no tienen definidos los tiempos de retiro aplicando sólo formulaciones de reconocida eficacia (Calvinho).

Desde hace ya más de diez años los programas de control de antibióticos en leche han adquirido mayor relevancia a nivel mundial, notándose una importante disminución en el número de muestras positivas a la presencia de estos compuestos, lo que demuestra la eficacia de estos programas.

La inclusión de esta prueba en el control de calidad de la materia prima por parte de esta empresa en particular, ha logrado mantener niveles de antibióticos inferiores al $1 \%$ anual desde la fecha. Resulta significativo justamente monitorear el momento del incremento en el control de calidad de la materia prima, mucho más que lo que en realidad sucede cuando el programa ya está establecido. Por ejemplo, en 1960 en los Estados Unidos la frecuencia de carros- cisterna positivos a residuos de antibióticos fue del 5\%, comparado con el $0.04 \%$ en 1994, sin embargo en otro estudio realizado en leche pasteurizada entre 1994 y 1997 se reportaron porcentajes sin mucha variación de muestras positivas de $0.063 \%$ en $1994,0.0101 \%$ en $1995,0.0106 \%$ en 1996 y $0.093 \%$ en 1997 (Philpot, 2004).

La aplicación de pruebas rápidas para medición de éste tipo de contaminación es de gran valor en leche, teniendo en cuenta su carácter perecedero como materia prima. Además, el tiempo consumido por prueba (seis minutos), es mucho más eficiente, rápida y específica que los métodos microbiológicos tradicionales, y mucho más barata que la aplicación de métodos cromatográficos como HPLC, que elevaría considerablemente los costos de producción. Para la industria láctea, desde el punto de vista práctico, no tiene utilidad conocer exactamente cuál es el antibiótico presente y su concentración exacta en la materia prima. Por otra parte, según Calderón, Rodríguez y Vélez, 2007, en un estudio realizado en Colombia, se encontró que los ganaderos conocían el concepto de inhibidores, y cuando la leche era comprada por empresas que pagan por calidad y realizan análisis para su determinación descartan esta leche; sin embargo, la venden con inhibidores, cuando los agentes comercializadores no realizan los respectivos controles, es decir no descartan esta leche.
Al parecer se trata de una mala práctica de manejo del alimento bastante diseminada en el ámbito lechero

Los valores de LMR de residuos de antibióticos en alimentos de origen animal, fueron finalmente establecidos en México en 2018, después de 24 años sin actualización, y representan el camino para lograr cumplir este importante indicador higiénico- sanitario (SADER- SENASICA, 2020).

Tres de los llamados eslabones de la cadena de la leche se ven afectados en diferente grado por la contaminación de la leche con residuos de antibióticos: el productor primario, la industria procesadora y finalmente el consumidor. Para el productor primario, la presencia de antibióticos trae aparejadas serias dificultades económicas, como la obtención de un precio más bajo por su producto, el riesgo de la revocación de su licencia de entrega a la industria, multas y pérdidas de volumen por leche no vendida, y finalmente un deterioro de la imagen del productor (Philpot, 2004), pero también se sabe que los ganaderos conocen el concepto de inhibidores; cuando la leche es comprada por empresas que vigilan la calidad del producto, ellos descartan las leches, sin embargo, la venden con antibióticos cuando los compradores no realizan controles (Máttar, Calderón, Sotelo, Sierra y Tordecilla, 2009).

En este caso particular, la empresa que sirvió como base para realizar el presente estudio tomó la decisión final de no aceptar leche positiva a antibióticos. La medida de control por parte de las empresas ha redundado en una solución parcial del problema de la incidencia de residuos de antibióticos en la leche, ya que existe evidencia de que en muchas ocasiones la industria láctea en México envasa y comercializa como leche fluida la leche cruda que se detecta como positiva a la presencia de antibióticos una vez acopiada (Ramírez et al, 2001, Noa-Lima, Noa, González, Landeros y Reyes, 2009).

En la legislación de la mayoría de los países, los inhibidores (antibióticos) son los únicos residuos químicos antropogénicos que se encuentran incluidos en los programas de control sistemático de la calidad de la leche cruda (NMXF-719, NOM-243). Este control cumple un doble propósito: con fines reguladores, y por parte de la industria láctea para el pago de la leche cruda. Si bien el beneficio logrado mediante programas de vigilancia en países desarrollados es innegable, en países con recursos económicos limitados como México, al decir de varios autores, la vigilancia constante es, a la fecha, utópica (Sumano y Ocampo, 2006), por lo que la solución completa de la problemática sólo sería posible dando un seguimiento al mismo por parte no sólo de las empresas lácteas, sino por la autoridades de salud competentes.

\section{Conclusión}

Se observó una disminución significativa en la detección de residuos de antibióticos $\beta$ - lactámicos y tetraciclinas desde el inicio de la aplicación del estudio, en la leche cruda acopiada por la planta pasteurizadora en el Estado de Jalisco, propor- 
cional al incremento en el número de tanques de leche analizados, lo que contribuyó a mejorar la calidad higiénicosanitaria de la leche cruda de vaca acopiada en el Estado de Jalisco.

\section{Literatura citada}

- AOAC. Official Methods of Analysis of AOAC International. 18th Edition. Rev. 1. 2006. Edited by: William Horwitz and Latimer, G. W., Gaithersburg, MD. 33.2.37.

- Calderón R. A, Rodríguez R, Vélez R S, 2007. Evaluaciónde la calidadde leches en cuatroprocesadoras de quesosen el municipio deMontería, Colombia Rev. MVZCórdoba 12 (1): 912- 920,Disponible en https://revistamvz.unicordoba.edu.co/article/view/435/503

- Calvinho, L. Actualización en tratamiento antibiótico de mastitis. Disponible en http://www.aprocal.com.ar/wpcontent/uploads/ACTUALIZACIONENTERAPIA2.htm.p df

- FIL/IDF. 1991: Yogurt Inhibitor Test in Bulletin of the International Dairy Federation $N^{\circ}$ 258/1991: Detection and Confirmation of inhibitors in milk and milk products. 2nd Edition. Brussels (Belgium). 46- 48.

- Kellnerová, E., Navrátilová, P. and Borkovcová I. 2014. Effect of pasteurization on the residues of tetracyclines in milk. ACTA VET. BRNO, 83: S21-S26; doi:10.2754/avb201483S10S21. Disponible en https://core.ac.uk/download/pdf/207103681.pdf

- Kurjogi M, Issa Mohammad YH, Alghamdi S, Abdelrahman M, Satapute P, Jogaiah S .2019 Detection and determination of stability of the antibiotic residues in cow's milk. PLoS ONE 14(10): e0223475. Disponible en https://doi.org/10.1371/journal. pone.0223475

- LEY GENERAL DE SALUD Nueva Ley publicada en el Diario Oficial de la Federación el 7 de febrero de 1984. TEXTO VIGENTE. Últimas reformas publicadas DOF 24-01-2020 Disponible en http://www.diputados.gob.mx/LeyesBiblio/pdf/142_2401 20.pdf

- Máttar, Salim, Alfonso Calderón, Diana Sotelo, Mónica Sierra y Gladys Tordecilla. 2009. Detección de Antibióticos en Leches: Un Problema de Salud Pública Rev. salud pública. 11 (4): 579-590

- NMX-F-700-COFOCALEC-2012. Norma MexicanaSistema producto leche- alimento - lácteo - leche cruda de vaca - Especificaciones Fisicoquímicas, Sanitarias y Métodos de Prueba. Organismo Nacional de Normalización del COFOCALEC. Disponible en http://www.cofocalec.org.mx/catalogo/por_clave

- NMX-F-719-COFOCALEC-2008. Norma Mexicana. Sistema- producto leche. Alimentos. Lácteos. Detección de inhibidores bacterianos en leche-Métodos de prueba rápidos. Disponible en http://www.cofocalec.org.mx/catalogo/por_clave

- Noa M, Ruvalcaba S. 2011: Ciencia de la Leche: una visión integral para México. Editorial Universitaria Universidad de Guadalajara.

- Noa-Lima, Elizabeth, Noa, M., González Delia, Landeros, Patricia, Reyes, Waldina. 2009. Evaluación de la presencia de residuos de antibióticos y quimioterapéuticos en leche en Jalisco, México. Rev. Salud Anim.. 31(1): 29- 33. Disponible en http://scielo.sld.cu/scielo.php?

script=sci_arttext\&pid=S0253-570X2009000100006

- NOM-155-SCFI-2012. Norma Oficial Mexicana. LecheDenominaciones, especificaciones fisicoquímicas, información comercial y métodos de prueba. Disponible en

http://www.dof.gob.mx/normasOficiales/4692/seeco/seec o.htm.

- NOM-243-SSA1-2010, NORMA Oficial Mexicana Productos y servicios. Leche, fórmula láctea, producto lácteo combinado y derivados lácteos. Disposiciones y especificaciones sanitarias. Métodos de prueba. Disponible en http://dof.gob.mx/normasOficiales/4156/salud2a/salud2a. htm.

- Philpot, W. N. 2004. Calidad de la leche y control de la mastitis: pasado, presente y futuro; Acontecer Lechero No22: 56- 59.

- PLM. 2019. Prontuario de Especialidades Veterinarias: Farmacéuticas, Biológicas, y Nutricionales. PLM México. Disponible en https://www.diccionarioveterinarioplm.com/

- Ramírez, Acacia, R. Gutiérrez, C. González, 1. Escobar, G. Castro, G. Días, G. Díaz y M. Noa. 2001. Detección de Antibióticos en Leche Comercializada en la Ciudad de México: Rev. Salud Anim. 23(1): 37- 41.

- Roca, L. Villegas, M. L. Kortabitarte, R. L. Althaus, and M. P. Molina. 2011. Effect of heat treatments on stability of $\beta$-lactams in milk J. Dairy Sci. 94 :1155- 1164 doi: 10.3168/jds.2010-3599. Disponible en https://www.researchgate.net/publication/49852889_Effe ct_of_heat_treatments_on_stability_of_b-

lactams_in_milk

- SADER/ SENASICA. 2020. Secretaría de Agricultura y Desarrollo Rural/ Servicio Nacional de Sanidad, Inocuidad y Calidad Agroalimentaria. Límites máximos de residuos tóxicos y contaminantes. TABLA DE LÍMITES MÁXIMOS DE RESIDUOS 2020. Disponible https://www.gob.mx/senasica/documentos/limitesmaximos-de-residuos-toxicos-y-contaminantes.

- Sumano,H.S. y L.Ocampo. 2006. Farmacología Veterinaria. Tercera Edición. McGraw- Hill Interamericana, Healthcare Group, México, D.F. 192. 819- 838 . 